\title{
Dharavi Slums (Mumbai, India): The Petri Dish of COVID-19 Herd Immunity
}

\author{
Zameer Shervani, Deepali Bhardwaj, and Roma Nikhat
}

\section{ABSTRACT}

\begin{abstract}
The first and second serosurvey results of COVID-19 antibodies in Dharavi and Cuffe Parade slums (Mumbai, India) were studied. The seroprevalence data of the slums explained the zero novel coronavirus cases reported in the slum dwellers verified first in real on the ground the concept of much needed herd immunity against the pandemic. First serosurvey data showed that more than half $(57 \%)$ of the population of Dharavi was infected with the virus. The antibodies were waned off in two month time and diminished to $45 \%$, as found in the second survey conducted after two months of the first survey. The antibodies prevalence was highest at $75 \%$ in Cuffe Parade slums of Mumbai. Initially, Dharavi slums were a hotspot of novel coronavirus which later became nearly a no-new infection zone. The herd immunity acquired in Dharavi residents stopped the new infection. One million Dharavi slum dwellers living in a crowded semi-isolated cluster mimic the petri-dish model of a human population which is now a near COVID-19 free zone due to the presence of antibodies in the residents. The infection rate in Maharashtra, India, and Dharavi slums was compared. The concept of herd immunity that occurred on the ground in real has been first established. The research is very useful to vaccinate populations to eradicate the pandemic from the globe.
\end{abstract}

Keywords: COVID-19; Herd immunity; CIVID-19 infections; serosurvey data; Dharavi slums.

\section{INTRODUCTION}

The second wave [1]-[6] of COVID-19 infection in India has done huge damage to the health and lives of the people. India recorded [7] 408,323 new COVID-19 cases on April 30, 2021 and became the first country to register more than 400,000 infections in a single day. Ten states of India: Maharashtra, Delhi, Karnataka, Kerala, Uttar Pradesh, Tamil Nadu, West Bengal, Andhra Pradesh, Rajasthan, and Bihar registered [8] 73.71\% of India`s new cases. On April 21, 2021, India surpassed [9] the US's highest number $(307,516)$ of cases recorded in a day on January 8, 2021. Many believed a more transmissible variant B.1.617 [10] of the novel coronavirus in India is the reason for large number of infections. The variant has B.1.617.1, B.1.617.2, and B.1.617.3 sub-lineages. In Japan, a total of 21 cases of the Indian variants have been detected. A double mutant variant in spike protein has two genetic changes. India has detected a triple mutant variant also. Indian variant is responsible for $60 \%$ of new infections. The genetic changes occurring in the spike proteins of the virus reduces antibodies neutralization by the vaccines. The three UK, Brazil, and South African variants have increased transmissibility and cause severe diseases leading to more deaths. The Indian variant has three mutations named L452R, P681R, and E484Q. L452R has increased transmissibility and reduced neutralization by monoclonal antibody treatment and vaccines. $\mathrm{L} 452 \mathrm{R}$ variant can bypass HLA (human leukocyte antigen)-24-restricted cellular immunity. $60 \%$ of Japanese and about $20 \%$ of Caucasians (US) have HLA-24 type of white blood corpuscle. For this reason, a large number of Japanese could be susceptible to the Indian L452R strain. L452R mutation has increased protein stability, viral infectivity, and viral replication is the threat of the pandemic. However, the mRNA vaccine developed by Pfizer-BioNTech is effective against Indian mutation. Surprisingly, the low COVID-19 infection rate in Japan and other East Asian countries (China, South Korea, Taiwan) is because of X-factors of using masks outdoors and genetic and immunological characteristics of the population. In the Indian variant, the N501Y segment is lacking thus, the variant could not be detected by polymerase chain reaction (PCR) and can only be identified by genome sequencing.

In the very recently published articles [11], [12], the onset of the second wave of the pandemic in India has been explained by the newly established "Health Index Theory". We have covered the pandemic aspect [13], vaccine development [14], and application of supercomputer [15] in controlling the COVID-19 disease. Article [16] described the possibility of virus transmission from pets to humans and vice versa. The novel coronavirus viability in different mediums and sanitization methods have been given in another article 
[17]. The present research article has verified first the herd immunity concept aimed at eradicating the COVID-19 pandemic from the globe. Antibodies developed in the population of Dharavi slums (Mumbai, India) slowed down and nearly stopped the spread of novel coronavirus infection in the locality. The daily infection rate and serosurvey results in the slums have been studied. Dharavi slums cluster spread in 2.5 kilometre $^{2}$ [18] area inhabited by a dense population of one million [19], [20] dwellers is good to imitate the petridish model of novel coronavirus transmission in a semiisolated locality. 10-12 persons on average live and share a tiny 10 feet $^{2}$ area room. The rooms are connected by narrow lanes and dwellers share common toilets and water taps. Considering the slums isolated cluster as a large petri-dish of population, the COVID-19 epidemiology has been studied. This is an example of a virus spread in a semi-closed and crowded place. The infection rate in the slums has been investigated versus the seroprevalence in the population. The research is very useful to test the herd immunity concept for vaccination in India and other parts of the world.

\section{RESULTS AND DISCUSSION}

\section{A. Serosurvey data of Dharavi and Cuffe Parade slums}

IgG SARS-CoV-2 antibodies in the blood serum of residents and healthcare workers of Mumbai slums (Dharavi and Cuffe Prade) and buildings have been analyzed. Figure 1 shows the results of the first and second serosurvey of Dharavi slums conducted in July-August and October 2020, respectively. The seroprevalence data recorded in October 2020 in Cuffe Prade slums have also been shown in Figure 1. Figure 1 shows that 57\% [21] people living in Dharavi slums were infected with the SARS-CoV-2 virus as per the first serosurvey conducted in July-August 2020. If more than half of the population tests positive for the virus antibodies is enough for herd immunity. The presence of protective antibodies in the blood of an individual will stop the infection. The people who develop antibodies will work as a wall against infecting others, thus stopping the virus transmission. Upon developing the herd immunity, the daily new infection curve will be flattened and subsequently start decreasing to verify the concept of COVID-19 pandemic herd immunity in slums. Only $16 \%$ (Fig. 1) of residents living outside slums in buildings were exposed to the virus due to possible physical distancing and better air ventilation compared to semichamber like slum houses.

The serosurvey conducted in October 2020 (second survey) showed that 45\% [22], [23] (Fig. 1) of the population in the Dharavi slums had seroprevalence witnessed a decrease by $12 \%$ as compared to the first survey. The sero prevalence in buildings was $18 \%$ compared to $16 \%$ in the first survey. The decrease in the seropositivity in the second survey is the reason of concern if the antibodies in asymptomatic or symptomatic patients have not waned off over time. The sero prevalence was $27 \%$ in healthcare workers in both the rounds of the survey indicated that staff attending the residents used non-pharmaceutical interventions of masks, social distancing, and hand hygiene. Serosurvey conducted [24] in Cuffe Parade slums in Mumbai in October 2020 found surprisingly $75 \%$ population positive as shown in Fig. 1. This was the highest so far reported in India. Seroprevalence (Fig. 2) in women and men was 79.3 and $70.8 \%$, respectively in October survey in Cuffe Parade slums of Mumbai. The age distribution of seropositive patients was 18-40, 40-60, and over 60 years of age had seroprevalence of 78,74 , and $76 \%$, respectively. The seroprevalence in women and men of first (Fig. 3) and second (Fig. 4) serosurvey have been shown. Antibodies prevalence in women is more than men in both the surveys showing that the against same SARS-CoV-2 viral load, antibodies formation is more in women than men. The overall antibodies data are very useful to vaccinate the population to eradicate the pandemic.

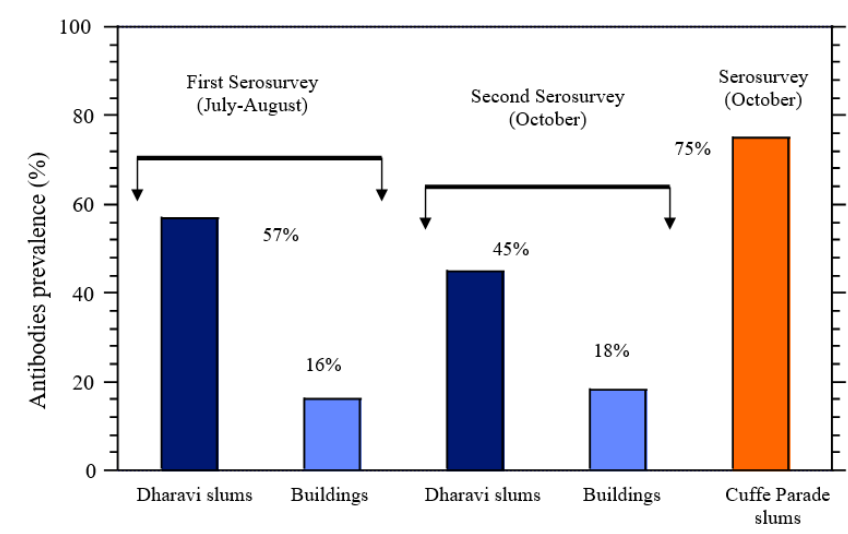

Fig. 1. Serosurvey results of Mumbai slums and buildings in 2020.

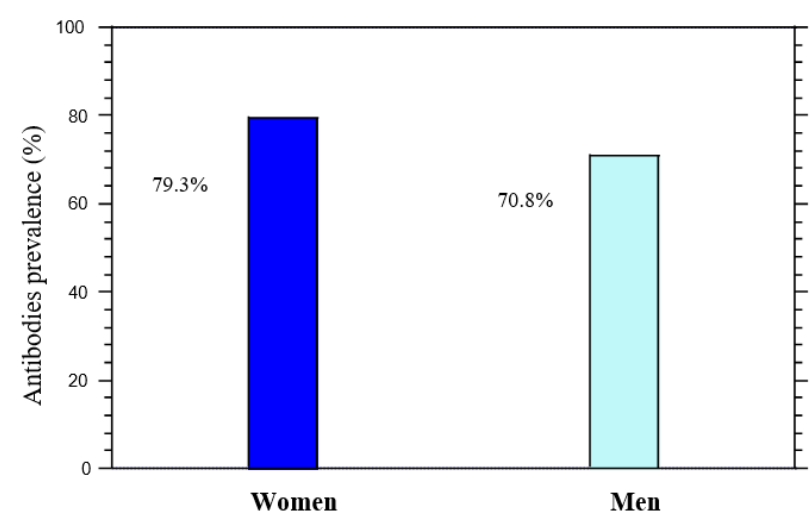

Fig. 2. Serosurvey results of men and women dwellers of Cuffe Parade slums (Mumbai) conducted in the month of October 2020.

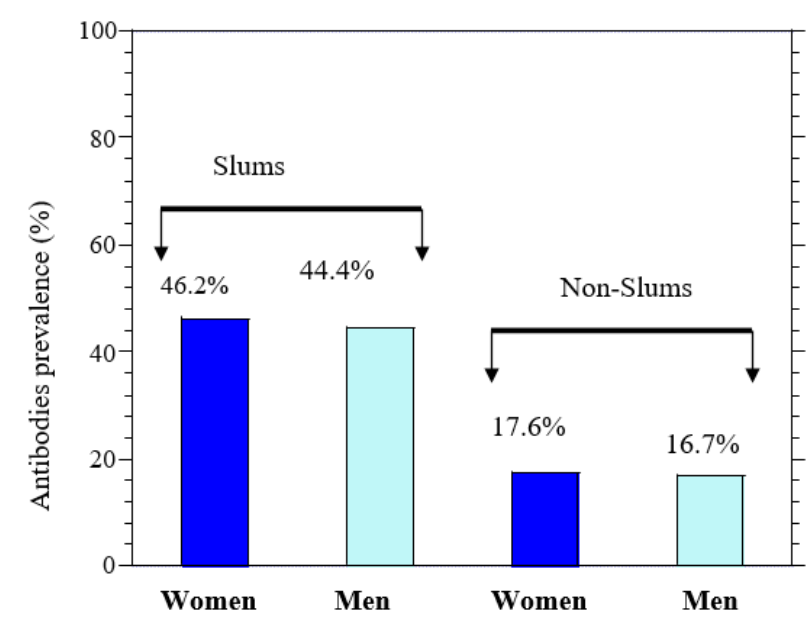

Fig. 3. Second serosurvey results of women and men dwellers of Dharavi slums and non-slums (Mumbai) conducted in month of July-August 2020. 


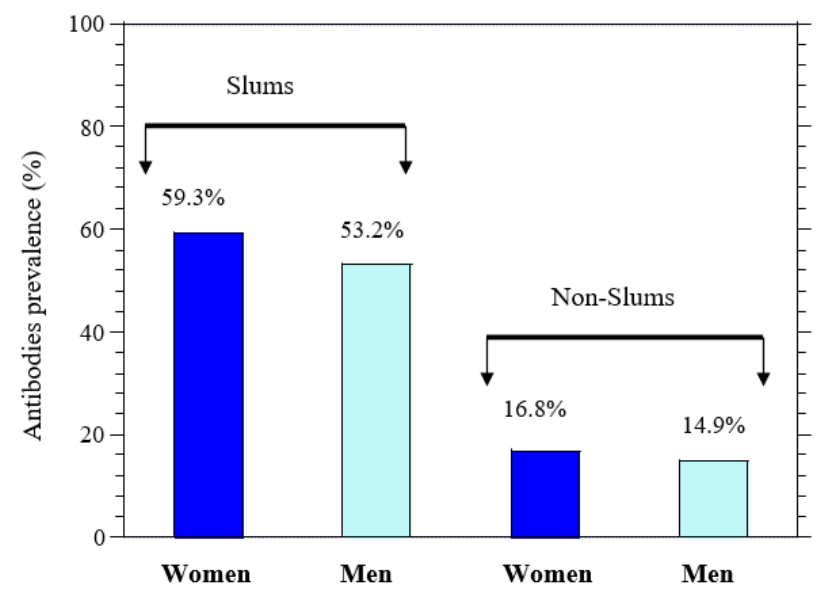

Fig. 4. First serosurvey results of women and men dwellers of Dharavi slums and non-slums (Mumbai) conducted in month of July-August 2020.

\section{B. The dynamics of Novel Coronavirus Spread in Dharavi Slums}

Fig. 5, 6, and 7 compare the dynamics of the virus spread in Dharavi slums, India, and Maharashtra. References [25][45] were used to construct Fig. 5, 6, and 7.

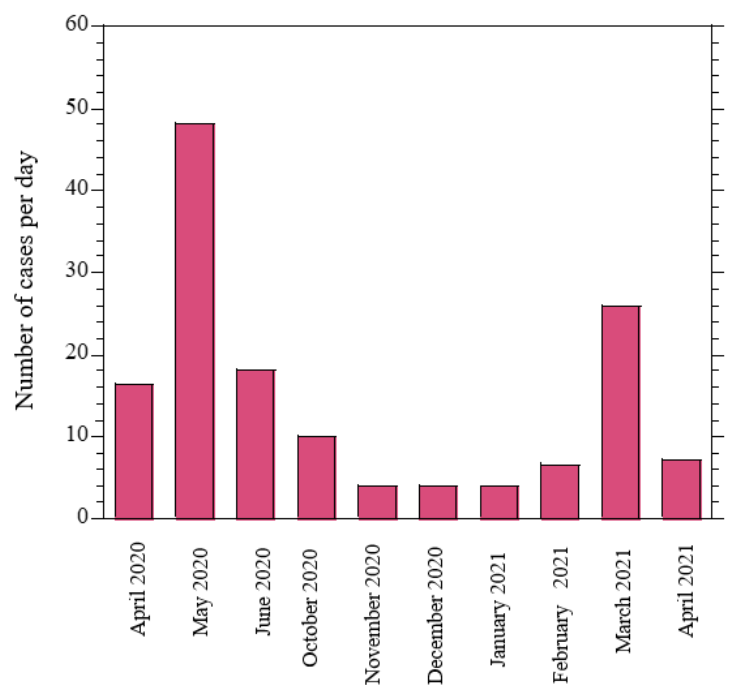

Fig. 5. Number of COVID-19 cases per day on average in the month shown in Dharavi slums.

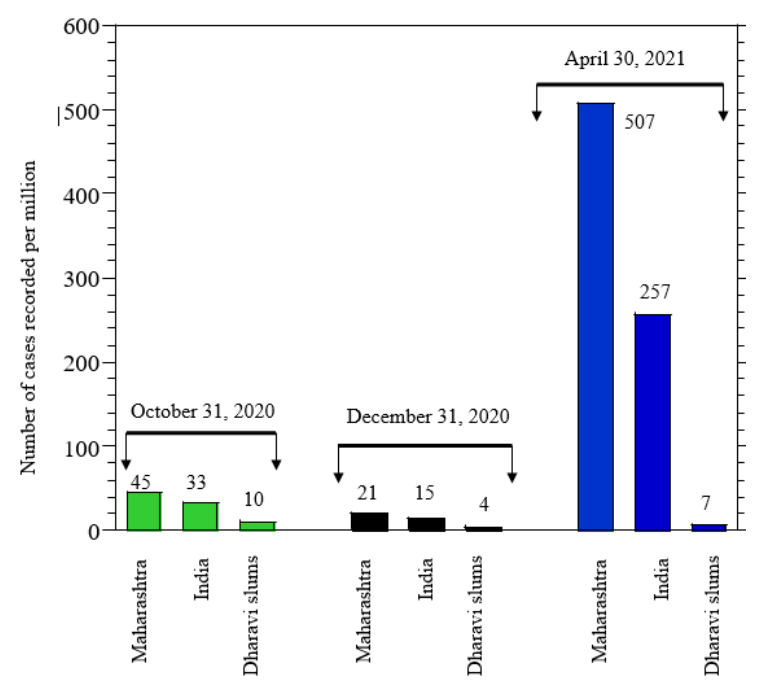

Fig. 6. Comparison of the number of COVID-19 cases recorded in one day on the date mentioned in Maharashtra, India, and Dharavi slums.

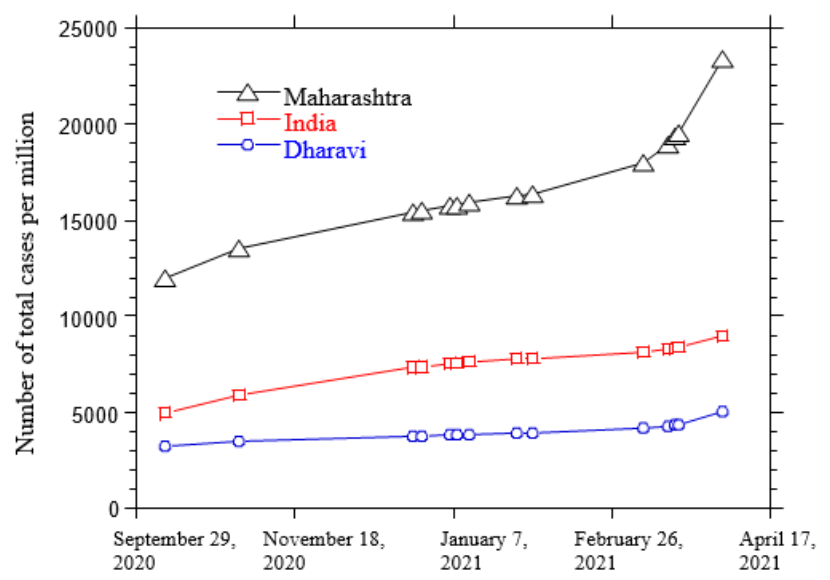

Fig. 7. Comparison of COVID-19 caseload (cases per million) of Dharavi slums, Maharashtra, and India.

In the initial stage of the COVID-19 pandemic, Dharavi slums were the hotspot as a large number (up to $>80$ cases per million) of cases [20] were recorded in a single day on some days in the period (April 25, 2020-May 15, 2020) whereas in India just 1-3 cases per million were registered in a day during the above period. Fig. 5 shows that 16, 48, and 18 cases per million were recorded on an average in a day in April, May, and June 2020, respectively in Dharavi slums. The number of infections in Dharavi peaked [20] in the last week of April and the first two weeks of May 2020 then subsequently started decreasing. The decrease was sharper in the last week of May and the decline continued till June 2020. The average number of cases remained in a single digit in November and December 2020 and January, February, and April 2021. Fig. 6 has bars showing the number of new cases recorded in Maharashtra, India, and Dharavi slums on the date mentioned. The number of cases in Dharavi remained $\leq 10$ whereas they were more in Maharashtra and India. Also, Dharavi did not show a surge in infections in the second wave of infection on April 30, 2021. Fig. 7 compares the cumulative number of cases (caseload) per million of novel coronavirus for the period October 8, 2020 to April 2, 2021, recorded in the state of Maharashtra, India, and Dharavi slums. In the above period, the number of new novel coronavirus cases per million per day on average recorded were 10, 22, and 65 cases for Dharavi slums, India, and Maharashtra. Such a low number of new infection cases and the absence of a new wave of the pandemic in Dharavi slums are only possible when the population develops herd immunity. The first and second serosurveys showed that the seroprevalence in Dharavi was 57 and $45 \%$, respectively. The serosurvey conducted in October 2020 for Cuffe Parade slums showed the antibodies presence in $75 \%$ population (described in section II A). Dharavi residents too might have seropositivity of $70-80 \%$, enough to develop herd immunity.

\section{STATEMENTS}

The data and results in this article are very reproducible. Author (Zameer Shervani, Ph.D.) is Director of Food \& Energy Security Research \& Product Center, Sendai, Japan. Roma Nikhat contributed remotely. Authors have qualifications; Deepali Bhardwaj MBBS, MD, DVDL, M.Phil. and Roma Nikhat MCA, MBA. Correspondence detail: WhatsApp: +818033214889 . 


\section{REFERENCES}

[1] Coronavirus in India April 7 Highlights: Vaccine shortage in Maharashtra; night curfew in Punjab, The Indian Express, April 8, 2021.

[2] As India records 126, 260 new Covid-19 cases, curbs in more states, Hindustan Times, April 8, 2021.

[3] Abhishek Jha, Covid-19: Urban hot spots drive surge, in greater need of jab scale-up, Hindustan Times, April 8, 2021.

[4] India records 35,886 Covid-19 infections, highest in 102 days, Times of India, March 18, 2021.

[5] Shuja Asrar, India on brink of second covid wave? At least 4 states of India witness fresh surge, TIMESOFINDIA.COM, February 20, 2021.

[6] Shuja Asrar, Covid cases: Why Kerala may be a 'victim` of its own 'success`, TIMESOFINDIA.COM, February 12, 2021.

[7] India became first country in the world to report over 4 lakhs new cases on April 30, 2021, The Hindu, April 30, 2021

[8] Maharashtra, Delhi among 10 states that account for 73.71 per cent of new Covid cases: Government, The Times of India, May 1, 2021.

[9] Amit Bhattacharya, More global records fall: Daily Covid cases hit 4 lakh, April count 69 lakh, The Times of India, May 1, 2021.

[10] Osamu Tsukimori, A new virus variant is infecting millions in India. What could its spread mean for Japan? The Japan Times, May 1, 2021.

[11] Shervani Z, COVID-19 in Kerala: Health Index Theory, European Journal of Medical and Health Sciences, 3, 2021, 21-24.

[12] Shervani Z, COVID-19 in Kerala: The Dynamics of Spread and Health Index Theory, RAS Medical Science, 2021, 1, 1-3.

[13] Shervani Z, et al. SARS-CoV-2 Delayed Tokyo 2020 Olympics: Very Recent Advances in COVID-19 Detection, Treatment, and Vaccine Development Useful Conducting the Games in 2021. Advances in Infectious Diseases. 2020; 10: 56-66.

[14] Shervani Z, et al. COVID-19 Vaccine. Advances in Infectious Diseases. 2020;10: 195-210.

[15] Shervani Z, et al. World's Fastest Supercomputer Picks COVID-19 Drug. Advances in Infectious Diseases. 2020; 10: 211-225.

[16] Shervani Z, et al. Risk of SARS-CoV-2 Transmission from Humans to Pets and Vice Versa, European Journal of Medical and Health Sciences, 3(2021)34-38

[17] Shervani Z, et al. Viability of SARS-CoV-2 and Sanitization Methods. European Journal of Medical and Health Sciences, 3(2021)22-27.

[18] Jyotsna Kaushal and Pooja Mahajan, Asia`s largest urban slumDharavi: A global model for management of COVID-19, Cities, 111, 2021, 103097.

[19] Dharavi, From Wikipedia, The Free Encyclopedia.

[20] Niha Masih, How a packed slum in Mumbai beat back the coronavirus, as India's cases continue to soar, The Washington Post, July 31, 2020.

[21] Over Half of People in Mumbai's Slums Probably Infected with Coronavirus: Survey, The Wire Science, July 30, 2020.
[22] Second sero-survey indicates drop in COVID-19 infections in Mumbai slums, The New Indian Express, October 2, 2020.

[23] Krishna Kumar, Latest sero survey results come in as a dampener for $\mathrm{BMC}$ and Maharashtra government, The Economics Times, October 2, 2020.

[24] COVID-19 antibodies were found in 75 percent of serosurvey participants from five slums in Mumbai's Cuffe Parade, Firstpost, November 30, 2020.

[25] Chaitanya Marpakwar, Zero cases in Dharavi, first time since April 1, The Times of India, December 26, 2020.

[26] Chaitanya Marpakwar, Mumbai: Zero new Covid-19 cases in Dharavi second time, The Times of India, January 22, 2021.

[27] Zero daily Covid-19 cases in Mumbai's slum area of Dharavi for second time since pandemic started, Hindustan Times, January 23, 2021.

[28] 30 New Covid Cases in Mumbai's Dharavi, First Daily High In 6 Months, NDTV.com, March 19, 2021.

[29] Mumbai's Dharavi reports highest ever spike of 99 coronavirus cases, Business Standard, April 8, 2021.

[30] Dharavi: How a slum city beat the virus, India Today, February 8, 2021.

[31] At 18, Dharavi reports highest daily Covid cases since October, India Today, March 8, 2021.

[32] COVID-19: Mumbai's Dharavi reports 21 cases, highest since October last year, The Free Press Journal, March 16, 2021.

[33] Mumbai: Mahim has more active coronavirus cases than Dharavi, Dadar combined, Mumbai Mirror, January 6, 2021.

[34] Mumbai: Dharavi reports just one new COVID-19 case today; active cases drop to 16, Mumbai Mirror, January 8, 2021.

[35] Coronavirus India Updates: Tally Tops 81 Lakh; Dharavi Reports Four New Cases, Bloomberg, October 31, 2020.

[36] Mumbai's slum area Dharavi records 8 new COVID-19 cases, Times of India, October 8, 2020.

[37] Mumbai's Dharavi records four new COVID-19 cases, Times of India, January 5, 2021.

[38] Mumbai: Dharavi reports 29 new cases on Friday, Hindustan Times, March 20, 2021.

[39] Mumbai: Covid-19 cases in Dharavi cross 5,000 mark; 694 active, Times of India, April 2, 2021.

[40] COVID-19 Tracker: Dharavi reports 99 new positive cases in the last 24 hours, Mumbai Mirror, April 8, 2021.

[41] Dharavi sees lowest new cases in 49 days, The Indian Express, April 28, 2021.

[42] Coronavirus (COVID-19) - Google News (JHU CSSE COVID-19 DATA).

[43] Our world in data COVID-19.

[44] Indiaonlinepages.com.

[45] populationu.com/in/uttar-pradesh-population. 